\title{
Synthesis of Pyrazine Substituted 1,3,4-Thiadiazole Derivatives and Their Anticonvulsant Activity
}

\author{
Kikkeri P. Harish, ${ }^{1}$ Kikkeri N. Mohana, ${ }^{1}$ and Lingappa Mallesha ${ }^{2}$ \\ ${ }^{1}$ Department of Studies in Chemistry, University of Mysore, Manasagangotri, Mysore 570 006, India \\ ${ }^{2}$ PG Department of Chemistry, JSS College of Arts, Commerce and Science, Ooty Road, Mysore 570025, India \\ Correspondence should be addressed to Kikkeri N. Mohana; drknmohana@gmail.com
}

Received 1 April 2013; Revised 13 September 2013; Accepted 13 September 2013

Academic Editor: Ashraf Aly Shehata

Copyright (c) 2013 Kikkeri P. Harish et al. This is an open access article distributed under the Creative Commons Attribution License, which permits unrestricted use, distribution, and reproduction in any medium, provided the original work is properly cited.

The synthesis of new pyrazine substituted 1,3,4-thiadiazole derivatives was carried out in good yield by the reaction of pyrazine substituted 1,3,4-thiadiazoles with various sulfonyl chlorides. A chemical structure of all the new compounds was confirmed by ${ }^{1} \mathrm{H}$ NMR and mass spectral data. The new compounds were screened for their anticonvulsant activity against maximal electroshock (MES) seizure method. Rotarod method was employed to determine the neurotoxicity. Few compounds showed significant changes in anticonvulsant activity. The same compounds showed no neurotoxicity at the maximum dose administered (100 mg/kg).

\section{Introduction}

Epilepsy has been recognized as a neurological disorder, affecting a large section of people across the world. The word epilepsy usually describes a group of common chronic neurological disorders characterized by recurrent unprovoked seizures due to excessive neuronal firing or synchronous neuronal activity in the brain $[1,2]$. Seizures may vary from the briefest lapses of attention or muscle jerks to severe and prolonged convulsions [3]. The maximal electroshock (MES) test is a predictor of compounds that are active against seizures [4]. The anticonvulsant drug design is based on the presumption that at least one phenyl or similar aromatic group in close proximity to two electron donor atoms in the compound is required for the activity in $\operatorname{MES}[5,6]$. Newer drugs such as flupirtine [7], topiramate [8], zonisamide [9], and vigabatrin [10] have emerged as promising anticonvulsants.

Pyrazines and its derivatives play an important role in the drug discovery realm. In particular the structural analogue of purines derivatives presents various pharmacological activities such as antibacterial [11], anti-inflammatory [12], antidepressant [13], and antiproliferative activities [14]. Thiadiazoles exhibit a broad spectrum of biological effectiveness such as antiparkinsonism [11], antihistaminic [15], and antiasthmatic [16]. Thiazolidin-4-one derivatives are also known to exhibit diverse bioactivities such as anticonvulsant [17], antidiarrheal [18], antihistaminic [19], antidiabetic [20], cardioprotective [21], and anticancer [22]. Similarly, 2,5-disubstituted 1,3,4thiadiazoles also display wide spectrum of activities such as antibacterial [23] and anticonvulsant [24]. In the present study, a series of new pyrazine substituted 1,3,4-thiadiazole derivatives $7(\mathbf{a}-\mathbf{o})$ have been synthesized and their anticonvulsant effects are determined through maximal electroshock (MES) seizure test.

\section{Materials and Methods}

2.1. Chemistry. Melting range was determined by Veego Melting Point VMP III apparatus. Elemental analyses were recorded on VarioMICRO superuser V1.3.2 Elementar. ${ }^{1} \mathrm{H}$ NMR spectra were recorded on Bruker DRX-500 spectrometer at $400 \mathrm{MHz}$ using DMSO- $\mathrm{d}_{6}$ as solvent and TMS as an internal standard. Mass spectral data were obtained by LC/MSD Trap XCT. Silica gel column chromatography was performed using Merck 7734 silica gel (60-120 mesh) and Merck-made TLC plates. 
2.1.1. Preparation of 1,3,4-Thiadiazole-2-ylamine (2). Thiosemicarbazide $(1,50.0 \mathrm{~g}, 0.5486 \mathrm{~mol})$ was taken in $100 \mathrm{~mL}$ formic acid and the reaction was stirred at room temperature for $1 \mathrm{hr}$. The reaction was cooled and $100 \mathrm{~mL}$ conc. hydrochloric acid was added. Reaction completion was monitored by TLC. The reaction was cooled to $0^{\circ} \mathrm{C}$ and basified with ammonium hydroxide solution. The solid formed was filtered, washed with water, and dried to yield the above compound as off white solid (Yield: $45.50 \mathrm{~g}, 82 \%) .{ }^{1} \mathrm{H}-$ NMR (400 MHz, DMSO-d 6 ): $\delta 4.21$ (s, br, 2H), 9.31 (s, 1H). MS (ESI) $m / z: 102.14$.

2.1.2. Preparation of 5-Bromo-1,3,4-thiadiazol-2-amine (3). To a stirred solution of $2(40.0 \mathrm{~g})$, sodium acetate $(64.89 \mathrm{~g})$ in $200 \mathrm{~mL}$ acetic acid (4.0 vol) at $10^{\circ} \mathrm{C}$, bromine was added drop wise $(37.92 \mathrm{~g})$. The reaction was stirred at room temperature for 3 hours. The reaction was concentrated and basified with saturated sodium bicarbonate solution. The compound was extracted with ethyl acetate and the ethyl acetate layer was washed with water followed by brine, dried over sodium sulphate. The crude product was crystallized using ethyl acetate to yield the above compound as off white solid (Yield: $53.4 \mathrm{~g}, 75 \%) .{ }^{1} \mathrm{H}-\mathrm{NMR}\left(400 \mathrm{MHz}, \mathrm{DMSO}^{-\mathrm{d}_{6}}\right): \delta 4.21$ (s, br, $2 \mathrm{H})$. MS (ESI) $m / z: 181.10$.

2.1.3. Preparation of tert-Butyl 4-(5-amino-1,3,4-thiadiazole2-yl)piperazine-1-carboxylate (4). To a stirred solution of 3 $(25.0 \mathrm{~g}, 0.1388 \mathrm{~mol})$, potassium carbonate $(57.57 \mathrm{~g}, 0.4165$ $\mathrm{mol})$ in dimethyl formamide $(250 \mathrm{~mL})$ and mono boc piperazine $(31.03 \mathrm{~g}, 0.1666 \mathrm{~mol})$ were added and stirred the reaction for $10 \mathrm{hr}$ at room temperature. The reaction was quenched into ice water $(2.0 \mathrm{~L})$ and stirred at room temperature for $1 \mathrm{hr}$. Solid formed was filtered and dried to yield the above compound as off white solid (Yield: $33.6 \mathrm{~g}, 85 \%$ ). ${ }^{1} \mathrm{H}-\mathrm{NMR}\left(400 \mathrm{MHz}, \mathrm{DMSO}-\mathrm{d}_{6}\right): \delta 1.41(\mathrm{~s}, 9 \mathrm{H}), 3.10(\mathrm{t}, J=$ $4.80 \mathrm{~Hz}, 4 \mathrm{H}), 3.77(\mathrm{t}, J=5.36 \mathrm{~Hz}, 4 \mathrm{H}), 4.21(\mathrm{~s}, 2 \mathrm{H})$. MS (ESI) $\mathrm{m} / z: 286.03$.

\subsubsection{Preparation of tert-Butyl-4-(5-(pyrazine-2-carbox-} amido)-1,3,4-thiadiazol-2-yl)piperazine-1-carboxylate (5). To a stirred solution of $4(25.0 \mathrm{~g}, 0.0876 \mathrm{~mol})$, in dichloromethane $(250 \mathrm{~mL})$, triethyl amine $(26.59 \mathrm{~g}, 0.2628 \mathrm{~mol})$, pyrazine-2-carboxylic acid $(13.04 \mathrm{~g}, 0.1051 \mathrm{~mol})$ and TBTU $(33.75 \mathrm{~g}, 0.1051 \mathrm{~mol})$ were added. The reaction was stirred overnight at room temperature and the reaction completion was monitored by TLC. The reaction was washed with saturated bicarbonate solution followed by $1.0 \mathrm{~N} \mathrm{HCl}$, water, and brine. The organic layer was dried over sodium sulphate, concentrated, and crystallized using dichloromethane to yield the above compound as off white solid (Yield: $28.80 \mathrm{~g}$, 84\%). ${ }^{1} \mathrm{H}-\mathrm{NMR}\left(400 \mathrm{MHz}, \mathrm{DMSO}-\mathrm{d}_{6}\right): \delta 1.41(\mathrm{~s}, 9 \mathrm{H}), 3.23$ $(\mathrm{t}, J=3.76 \mathrm{~Hz}, 4 \mathrm{H}), 3.75(\mathrm{t}, J=4.40 \mathrm{~Hz}, 4 \mathrm{H}), 8.83(\mathrm{~d}, J=$ $2.00 \mathrm{~Hz}, 1 \mathrm{H}), 8.94(\mathrm{~d}, J=2.00 \mathrm{~Hz}, 1 \mathrm{H}), 9.25$ (s, $1 \mathrm{H}), 9.72$ (s, $2 \mathrm{H})$.

2.1.5. Preparation of N-(5-(Piperazine-1-yl)-1,3,4-thiadiazole2-yl)pyrazine-2-carboxamide hydrochloride (6). To a stirred solution of $5(20 \mathrm{~g}, 0.0687 \mathrm{~mol})$ in 1,4 dioxane at $0-5^{\circ} \mathrm{C}$,
$4 \mathrm{~N} \mathrm{HCl}$ in dioxane $(80.0 \mathrm{~mL})$ was added. The reaction was stirred at room temperature for $10 \mathrm{hr}$. The solid was filtered, washed with diethyl ether, and packed in air tight container to yield the above compound as pale yellow hygroscopic solid $(16.73 \mathrm{~g}, 90 \%) .{ }^{1} \mathrm{H}-\mathrm{NMR}\left(400 \mathrm{MHz}, \mathrm{DMSO}-\mathrm{d}_{6}\right): \delta 3.22(\mathrm{t}, J=$ $3.75 \mathrm{~Hz}, 4 \mathrm{H}), 3.73(\mathrm{t}, J=4.30 \mathrm{~Hz}, 4 \mathrm{H}), 8.83(\mathrm{~d}, J=2.00 \mathrm{~Hz}$, $1 \mathrm{H}), 8.94(\mathrm{~d}, J=2.00 \mathrm{~Hz}, 1 \mathrm{H}), 9.27$ (s, 1H), 9.72 (s, 2H). MS (ESI) $m / z: 292.05$.

2.1.6. General Procedure for the Synthesis of Pyrazine Substituted 1,3,4-Thiadiazole Derivatives $7(\boldsymbol{a}-\boldsymbol{o})$. To a mixture of 6 (1.0 eq) and triethyl amine (4.5 eq) in dichloromethane (10 volume), substituted sulphonyl chloride ( 2.4 eq) was added at $5-10^{\circ} \mathrm{C}$ and stirred overnight at room temperature. Reaction completion was confirmed through TLC. The reaction was poured into separating funnel, washed with water followed by brine solution, and dried over anhydrous sodium sulphate. Crude product was purified by crystallization using ethyl acetate to yield the thiadiazole substituted sulfonamide as off white to pale yellow solids.

(1) N-(5-(4-((1,l'-Biphenyl)sulfonyl)piperazine-1-yl)-1,3,4-thiadiazole-2-yl)pyrazine-2-carboxamide (7a). Yield: 70\% (White solid); ${ }^{1} \mathrm{H}-\mathrm{NMR}\left(400 \mathrm{MHz}, \mathrm{DMSO}-\mathrm{d}_{6}\right): \delta 3.18(\mathrm{t}, J=4.10 \mathrm{~Hz}$, $4 \mathrm{H}), 3.67(\mathrm{t}, J=5.02 \mathrm{~Hz}, 4 \mathrm{H}), 7.40-7.52(\mathrm{~m}, 3 \mathrm{H}), 7.64-7.87$ $(\mathrm{m}, 6 \mathrm{H}), 8.81(\mathrm{~d}, J=2.35 \mathrm{~Hz}, 1 \mathrm{H}), 8.90(\mathrm{~d}, J=2.45 \mathrm{~Hz}, 1 \mathrm{H})$, $9.26(\mathrm{~s}, 1 \mathrm{H}), 12.2$ (s, 1H). MS (ESI) $m / z: 508.6$. Anal. Calcd. for $\mathrm{C}_{23} \mathrm{H}_{21} \mathrm{~N}_{7} \mathrm{O}_{3} \mathrm{~S}_{2}$ : C, 54.42; H, 4.17; N, 19.32; Found: C, 54.13; H, $4.32 ; \mathrm{N}, 19.52 \%$.

(2) N-(5-(4-((2,4-Dimethylphenyl)sulfonyl)piperazine-1-yl)-1, 3,4-thiadiazole-2-yl)pyrazine-2-carboxamide ( $7 \boldsymbol{b})$. Yield: $72 \%$ (Off white solid); ${ }^{1} \mathrm{H}-\mathrm{NMR}\left(400 \mathrm{MHz}, \mathrm{DMSO}-\mathrm{d}_{6}\right): \delta 2.34(\mathrm{~s}$, $3 \mathrm{H}), 2.37(\mathrm{~s}, 3 \mathrm{H}), 3.21(\mathrm{t}, J=4.01 \mathrm{~Hz}, 4 \mathrm{H}), 3.68(\mathrm{t}, J=4.90 \mathrm{~Hz}$, $4 \mathrm{H}), 7.22(\mathrm{~s}, 1 \mathrm{H}), 7.42(\mathrm{~d}, J=6.35 \mathrm{~Hz}, 2 \mathrm{H}), 8.70(\mathrm{~d}, J=2.25 \mathrm{~Hz}$, $1 \mathrm{H}), 8.81(\mathrm{~d}, J=2.46 \mathrm{~Hz}, 1 \mathrm{H}), 9.16(\mathrm{~s}, 1 \mathrm{H}), 12.3$ (s, 1H). MS (ESI) $m / z: 460.60$. Anal. Calcd. for $\mathrm{C}_{19} \mathrm{H}_{21} \mathrm{~F}_{6} \mathrm{~N}_{7} \mathrm{O}_{3} \mathrm{~S}_{2}$ : C, 49.66; $\mathrm{H}$, 4.61; N, 21.34; Found: C, 49.75; H, 4.68; N, 21.46\%.

(3) N-(5-(4-(Methylsulfonyl)piperazine-1-yl)-1,3,4-thiadiazole -2-yl)pyrazine-2-carboxamide (7c). Yield: 72\% (White solid); ${ }^{1} \mathrm{H}-\mathrm{NMR}\left(400 \mathrm{MHz}, \mathrm{DMSO}-\mathrm{d}_{6}\right): \delta 2.35(\mathrm{~s}, 3 \mathrm{H}), 3.23(\mathrm{t}, J=$ $3.86 \mathrm{~Hz}, 4 \mathrm{H}), 3.72(\mathrm{t}, J=4.31 \mathrm{~Hz}, 4 \mathrm{H}), 8.83(\mathrm{~d}, J=2.00 \mathrm{~Hz}$, $1 \mathrm{H}), 8.94$ (d, $J=2.00 \mathrm{~Hz}, 1 \mathrm{H}), 9.27$ (s, 1H), 12.1 (s, br, 1). MS (ESI) $m / z$ : 370.40. Anal. Calcd. for $\mathrm{C}_{12} \mathrm{H}_{15} \mathrm{~N}_{7} \mathrm{O}_{3} \mathrm{~S}_{2}$ : C, 39.01; H, 4.09; N, 26.54; Found: C, 38.97; H, 3.96; N, 26.74\%.

(4) N-(5-(4-((2,5-Dichlorothiophen-3-yl)sulfonyl)piperazine1-yl)-1,3,4-thiadiazole-2-yl)pyrazine-2-carboxamide (7d). Yield: $74 \%$ (Off white solid); ${ }^{1} \mathrm{H}-\mathrm{NMR}(400 \mathrm{MHz}, \mathrm{DMSO}-$ $\left.\mathrm{d}_{6}\right): \delta 3.31(\mathrm{t}, J=4.08 \mathrm{~Hz}, 4 \mathrm{H}), 3.61(\mathrm{t}, J=3.84 \mathrm{~Hz}, 4 \mathrm{H}), 7.43$ $(\mathrm{s}, 1 \mathrm{H}), 8.83(\mathrm{~d}, J=2.20 \mathrm{~Hz}, 1 \mathrm{H}), 8.95(\mathrm{~d}, J=2.24 \mathrm{~Hz}, 1 \mathrm{H})$, 9.28 (s, 1H), 12.40 (s, 1H). MS (ESI) m/z: 505.50. Anal. Calcd. for $\mathrm{C}_{15} \mathrm{H}_{13} \mathrm{Cl}_{2} \mathrm{~N}_{7} \mathrm{O}_{3} \mathrm{~S}_{3}$ : C, 35.58; H, 2.59; N, 19.36; Found: C, $35.35 ; \mathrm{H}, 2.75 ; \mathrm{N}, 19.54 \%$.

(5) N-(Naphthalene-2-ylsulfonyl)-N-(5-(4-(naphthalene-2-ylsulfonyl)piperazine-1-yl)-1,3,4-thiadiazole-2-yl)pyrazine-2carboxamide $(7 \boldsymbol{e})$. Yield: $75 \%$ (Off white solid); ${ }^{1} \mathrm{H}-\mathrm{NMR}$ 
$\left(400 \mathrm{MHz}, \mathrm{DMSO}_{-} \mathrm{d}_{6}\right): \delta 3.14(\mathrm{t}, J=4.96 \mathrm{~Hz}, 4 \mathrm{H}), 3.57(\mathrm{t}, J=$ $5.36 \mathrm{~Hz}, 4 \mathrm{H}), 7.50-7.54(\mathrm{~m}, 2 \mathrm{H}), 7.67-7.79$ (m, 5H), 7.85-7.87 (m, $1 \mathrm{H}), 7.88-7.91(\mathrm{~m}, 1 \mathrm{H}), 8.09-8.24(\mathrm{~m}, 4 \mathrm{H}), 8.48(\mathrm{~s}, 1 \mathrm{H})$, $8.80(\mathrm{~d}, J=2.32 \mathrm{~Hz}, 1 \mathrm{H}), 8.92(\mathrm{~d}, J=2.44 \mathrm{~Hz}, 1 \mathrm{H}), 9.23(\mathrm{~s}, 1 \mathrm{H})$. MS (ESI) $m / z$ : 686.80. Anal. Calcd. for $\mathrm{C}_{32} \mathrm{H}_{27} \mathrm{~N}_{7} \mathrm{O}_{5} \mathrm{~S}_{3}$ : C, 55.43 ; H, 3.75; N, 14.60; Found: C, 55.54; H, 3.65; N, 14.42\%.

(6) N-(5-(4-((3,5-Dimethylisoxazol-4-yl)sulfonyl)piperazine1-yl)1,3,4-thiadiazole-2-yl) pyrazine-2-carboxamide ( $7 \boldsymbol{f})$. Yield: $73 \%$ (Off white solid); ${ }^{1} \mathrm{H}-\mathrm{NMR}(400 \mathrm{MHz}$, DMSO$\left.\mathrm{d}_{6}\right): \delta 2.34(\mathrm{~s}, 3 \mathrm{H}), 2.62(\mathrm{~s}, 3 \mathrm{H}), 3.22(\mathrm{t}, J=4.92 \mathrm{~Hz}, 4 \mathrm{H}), 3.58$ $(\mathrm{t}, J=5.28 \mathrm{~Hz}, 4 \mathrm{H}), 8.82(\mathrm{~d}, J=2.36 \mathrm{~Hz}, 1 \mathrm{H}), 8.93(\mathrm{~d}, J=$ $2.44 \mathrm{~Hz}, 1 \mathrm{H}), 9.26$ (s, 1H), 12.30 (s, 1H). MS (ESI) $m / z: 451.00$. Anal. Calcd. for $\mathrm{C}_{16} \mathrm{H}_{18} \mathrm{~N}_{8} \mathrm{O}_{4} \mathrm{~S}_{2}$ : C, 42.66; H, 4.03; N, 24.87; Found: C, 42.78; H, 4.30; N, 24.75\%.

(7) N-(5-(4-((3,5-Bis(trifluoromethyl)phenyl)sulfonyl)piperazine-1-yl)-1,3,4-thiazol-2-yl) pyrazine-2-carboxamide (7g). Yield: $72 \%$ (Off white solid); ${ }^{1} \mathrm{H}-\mathrm{NMR}(400 \mathrm{MHz}, \mathrm{DMSO}-$ $\left.\mathrm{d}_{6}\right): \delta 3.31(\mathrm{t}, J=4.08 \mathrm{~Hz}, 4 \mathrm{H}), 3.88(\mathrm{t}, J=4.96 \mathrm{~Hz}, 4 \mathrm{H}), 8.15$ (s, 1H), 8.23 (s, br, 2H), 8.71 (d, $J=2.16 \mathrm{~Hz}, 1 \mathrm{H}), 8.74(\mathrm{~s}, 1 \mathrm{H})$, $8.91(\mathrm{~d}, J=2.28 \mathrm{~Hz}, 1 \mathrm{H}), 9.47(\mathrm{~s}, 1 \mathrm{H})$. MS (ESI) $m / z: 566.40$. Anal. Calcd. for $\mathrm{C}_{19} \mathrm{H}_{15} \mathrm{~F}_{6} \mathrm{~N}_{7} \mathrm{O}_{3} \mathrm{~S}_{2}$ : C, 40.21; H, 2.66; N, 17.28; Found: C, 40.28; H, 2.54; N, 17.43\%.

(8) N-(5-(4-Tosylpiperazine-1-yl)-1,3,4-thiadiazole-2-yl)pyrazine-2-carboxamide (7h). Yield: $71 \%$ (Off white solid); ${ }^{1} \mathrm{H}-\mathrm{NMR}\left(400 \mathrm{MHz}, \mathrm{DMSO}-\mathrm{d}_{6}\right): \delta 2.24(\mathrm{~s}, 3 \mathrm{H}), 3.25(\mathrm{t}, J=$ $4.82 \mathrm{~Hz}, 4 \mathrm{H}), 3.78(\mathrm{t}, J=5.08 \mathrm{~Hz}, 4 \mathrm{H}), 7.75(\mathrm{~d}, J=8.58 \mathrm{~Hz}$, $2 \mathrm{H}), 8.12(\mathrm{~d}, J=8.13 \mathrm{~Hz}, 2 \mathrm{H}), 8.82(\mathrm{~d}, J=2.26 \mathrm{~Hz}, 1 \mathrm{H}), 8.93$ (d, $J=2.34 \mathrm{~Hz}, 1 \mathrm{H}), 9.23$ (s, 1H), 12.27 (s, 1H). MS (ESI) $m / z$ : 444.50. Anal. Calcd. for $\mathrm{C}_{18} \mathrm{H}_{19} \mathrm{~N}_{7} \mathrm{O}_{3} \mathrm{~S}_{2}$ : C, 48.53; $\mathrm{H}, 4.30 ; \mathrm{N}$, 22.01; Found: C, 48.67; H, 4.42; N, 22.31\%.

(9) N-(5-(4-((4-(tert-Butyl)phenyl)sulfonyl)piperazine-1-yl)1,3,4-thiadiazole-2-yl)pyrazine-2-carboxamide (7i). Yield: $73 \%$ (Off white solid); ${ }^{1} \mathrm{H}-\mathrm{NMR}\left(400 \mathrm{MHz}, \mathrm{DMSO}-\mathrm{d}_{6}\right): \delta$ $1.14(\mathrm{~s}, 9 \mathrm{H}), 3.41(\mathrm{t}, J=4.72 \mathrm{~Hz}, 4 \mathrm{H}), 3.93(\mathrm{t}, J=5.28 \mathrm{~Hz}, 4 \mathrm{H})$, $7.72(\mathrm{~d}, J=7.68 \mathrm{~Hz}, 2 \mathrm{H}), 7.99$ (d, $J=7.83 \mathrm{~Hz}, 2 \mathrm{H}), 8.80$ (d, $J=$ $2.27 \mathrm{~Hz}, 1 \mathrm{H}), 8.91$ (d, $J=2.44 \mathrm{~Hz}, 1 \mathrm{H}), 9.21$ (s, $1 \mathrm{H}), 12.23$ (s, $1 \mathrm{H})$. MS (ESI) $m / z$ : 486.50. Anal. Calcd. for $\mathrm{C}_{21} \mathrm{H}_{25} \mathrm{~N}_{7} \mathrm{O}_{3} \mathrm{~S}_{2}$ : C, 51.73; H, 5.17; N, 20.11; Found: C, 51.53; H, 5.32; N, 20.32\%.

(10) N-(5-(4-((3-(Trifluoromethyl)phenyl)sulfonyl)piperazine1-yl)-1,3,4-thiadiazole-2-yl)pyrazine-2-carboxamide

$(7 \boldsymbol{j})$. Yield: 73\% (Off white solid); ${ }^{1} \mathrm{H}-\mathrm{NMR}$ (400 MHz, DMSO$\left.\mathrm{d}_{6}\right): \delta 3.15(\mathrm{t}, J=4.40 \mathrm{~Hz}, 4 \mathrm{H}), 3.60(\mathrm{t}, J=5.20 \mathrm{~Hz}, 4 \mathrm{H})$, 7.90-7.94 (m, 1H), $8.00(\mathrm{~s}, 1 \mathrm{H}), 8.09-8.14(\mathrm{~m}, 2 \mathrm{H}), 8.78$ $(\mathrm{d}, J=2.26 \mathrm{~Hz}, 1 \mathrm{H}), 8.85(\mathrm{~d}, J=2.44 \mathrm{~Hz}, 1 \mathrm{H}), 9.23(\mathrm{~s}$, $1 \mathrm{H}), 12.23$ (s, 1H). MS (ESI) $\mathrm{m} / z$ : 498.50. Anal. Calcd. for $\mathrm{C}_{18} \mathrm{H}_{16} \mathrm{~F}_{3} \mathrm{~N}_{7} \mathrm{O}_{3} \mathrm{~S}_{2}$ : C, 43.28; $\mathrm{H}, 3.23$;, 19.63 ; Found: $\mathrm{C}$, 43.43; H, 3.43; N, 19.73\%.

(11) N-(5-(4-((4-Iodophenyl)sulfonyl)piperazine-1-yl)-1,3,4thiadiazole-2-yl)pyrazine-2-carboxamide (7k). Yield: $71 \%$ (Off white solid); ${ }^{1} \mathrm{H}-\mathrm{NMR}\left(400 \mathrm{MHz}, \mathrm{DMSO}-\mathrm{d}_{6}\right): \delta 3.06(\mathrm{t}$, $J=3.80 \mathrm{~Hz}, 4 \mathrm{H}), 3.45(\mathrm{t}, J=4.10 \mathrm{~Hz}, 4 \mathrm{H}), 7.68(\mathrm{~d}, J=7.05 \mathrm{~Hz}$, $2 \mathrm{H}), 7.86(\mathrm{~d}, J=6.75 \mathrm{~Hz}, 2 \mathrm{H}), 8.78(\mathrm{~d}, J=2.22 \mathrm{~Hz}, 1 \mathrm{H}), 8.92$ (d, $J=2.34 \mathrm{~Hz}, 1 \mathrm{H}), 9.24(\mathrm{~s}, 1 \mathrm{H}), 12.28(\mathrm{~s}, 1 \mathrm{H})$. MS (ESI) $m / z$ : 556.80. Anal. Calcd. for $\mathrm{C}_{17} \mathrm{H}_{16} \mathrm{IN}_{7} \mathrm{O}_{3} \mathrm{~S}_{2}$ : C, 36.63; H, 2.89; N, 17.59; Found: C, 36.53; H, 2.75; N, 17.64\%.

(12) N-(5-(4-((Cyclopropane)sulfonyl)piperazine-1-yl)-1,3,4thiadiazole-2-yl)pyrazine-2-carboxamide ( $7 l)$. Yield: 75\% (Off white solid); ${ }^{1} \mathrm{H}-\mathrm{NMR}\left(400 \mathrm{MHz}, \mathrm{DMSO}-\mathrm{d}_{6}\right): \delta 0.7-0.8(\mathrm{~m}$, $4 \mathrm{H}), 1.0-1.02(\mathrm{~m}, 1 \mathrm{H}), 3.26(\mathrm{t}, J=3.70 \mathrm{~Hz}, 4 \mathrm{H}), 3.72(\mathrm{t}, J=$ $4.28 \mathrm{~Hz}, 4 \mathrm{H}), 8.83(\mathrm{~d}, J=2.20 \mathrm{~Hz}, 1 \mathrm{H}), 8.94(\mathrm{~d}, J=2.32 \mathrm{~Hz}$, $1 \mathrm{H}), 9.23$ (s, 1H), 9.70 (s, 1H). MS (ESI) $m / z$ : 394.40. Anal. Calcd. for $\mathrm{C}_{14} \mathrm{H}_{17} \mathrm{~N}_{7} \mathrm{O}_{3} \mathrm{~S}_{2}$ : C, 42.52; $\mathrm{H}, 4.33 ; \mathrm{N}, 24.79$; Found: C, 42.43; H, 4.54; N, 24.86\%.

(13) N-(5-(4-((2-Nitro-4-(trifluoromethyl)phenyl)sulfonyl)piperazine-1-yl)-1,3,4-thiadiazole-2-yl)pyrazine-2-carboxamide $(7 m)$. Yield: $75 \%$ (Off white solid); ${ }^{1} \mathrm{H}-\mathrm{NMR}(400 \mathrm{MHz}$, DMSO $\left.-\mathrm{d}_{6}\right): \delta 3.31(\mathrm{t}, J=4.08 \mathrm{~Hz}, 4 \mathrm{H}), 3.88(\mathrm{t}, J=4.96 \mathrm{~Hz}$, $4 \mathrm{H}), 8.25$ (s, br, 2H), $8.65(\mathrm{~s}, 1 \mathrm{H}), 8.81(\mathrm{~d}, J=2.40 \mathrm{~Hz}, 1 \mathrm{H})$, $8.93(\mathrm{~d}, J=2.68 \mathrm{~Hz}, 1 \mathrm{H}), 9.26(\mathrm{~s}, 1 \mathrm{H}), 12.30$ (s, 1H). MS (ESI) $m / z$ : 543.50. Anal. Calcd. for $\mathrm{C}_{18} \mathrm{H}_{15} \mathrm{~F}_{3} \mathrm{~N}_{8} \mathrm{O}_{5} \mathrm{~S}_{2}$ : C, 39.71; $\mathrm{H}$, 2.78; N, 20.58; Found: C, 39.79; H, 2.65; N, 20.76\%.

(14) N-(5-(4-((4-Bromophenyl)sulfonyl)piperazine-1-yl)-1,3,4thiadiazole-2-yl)pyrazine-2-carboxamide (7n). Yield: $72 \%$ (Off white solid); ${ }^{1} \mathrm{H}-\mathrm{NMR}\left(400 \mathrm{MHz}, \mathrm{DMSO}-\mathrm{d}_{6}\right): \delta 3.16(\mathrm{t}$, $J=3.90 \mathrm{~Hz}, 4 \mathrm{H}), 3.55(\mathrm{t}, J=4.20 \mathrm{~Hz}, 4 \mathrm{H}), 7.71(\mathrm{~d}, J=7.25 \mathrm{~Hz}$, $2 \mathrm{H}), 7.83(\mathrm{~d}, J=7.65 \mathrm{~Hz}, 2 \mathrm{H}), 8.73(\mathrm{~d}, J=2.29 \mathrm{~Hz}, 1 \mathrm{H}), 8.90$ (d, $J=2.34 \mathrm{~Hz}, 1 \mathrm{H}), 9.28(\mathrm{~s}, 1 \mathrm{H}), 12.18(\mathrm{~s}, 1 \mathrm{H})$. MS (ESI) $m / z$ : 509.40. Anal. Calcd. for $\mathrm{C}_{17} \mathrm{H}_{16} \mathrm{BrN}_{7} \mathrm{O}_{3} \mathrm{~S}_{2}$ : C, 40.01; H, 3.16; N, 19.21; Found: C, 39.98; H, 3.32; N, 19.32\%.

(15) N-(5-(4-((4-(Trifluoromethyl)phenyl)sulfonyl)piperazine1-yl)-1,3,4-thiadiazole-2-yl)pyrazine-2-carboxamide (7o). Yield: $73 \%$ (Off white solid); ${ }^{1} \mathrm{H}-\mathrm{NMR}(400 \mathrm{MHz}, \mathrm{DMSO}-$ $\left.\mathrm{d}_{6}\right): \delta 3.16(\mathrm{t}, J=4.40 \mathrm{~Hz}, 4 \mathrm{H}), 3.68(\mathrm{t}, J=5.20 \mathrm{~Hz}, 4 \mathrm{H})$, $7.10(\mathrm{dd}, J=1.84,7.42 \mathrm{~Hz}, 2 \mathrm{H}), 8.09-8.14(\mathrm{~m}, 2 \mathrm{H}), 8.71$ $(\mathrm{d}, J=2.25 \mathrm{~Hz}, 1 \mathrm{H}), 8.82(\mathrm{~d}, J=2.44 \mathrm{~Hz}, 1 \mathrm{H}), 9.27$ (s, $1 \mathrm{H}), 12.21$ ( $\mathrm{s}, 1 \mathrm{H})$. MS (ESI) $m / z$ : 498.40. Anal. Calcd. for $\mathrm{C}_{18} \mathrm{H}_{16} \mathrm{~F}_{3} \mathrm{~N}_{7} \mathrm{O}_{3} \mathrm{~S}_{2}$ : C, 43.28; H, 3.23; N, 19.63; Found: C, 43.38; $\mathrm{H}, 3.42 ; \mathrm{N}, 19.60 \%$.

\subsection{Anticonvulsant Evaluation}

2.2.1. Animals. Male wistar rats procured from the National Institute of Nutrition, Hyderabad (190-220 g), were used in the present study. The animals were kept in individual cages for one week to acclimatize for the laboratory conditions. They were allowed to free access of water and food.

All the experimental procedures were carried out in accordance with Committee for the Purpose of Control and Supervision of Experiments on Animals (CPCSEA) guidelines. The study was reviewed and approved by the Institutional Animal Ethics Committee, G Pulla Reddy College of Pharmacy, Hyderabad, India.

2.2.2. Maximal Electroshock Seizure Model (MES). Maximal electroshock seizure model was used in the present study to evaluate the anticonvulsant activity of the compounds 
TABLE 1: Chemical structure and melting range of pyrazine substituted 1,3,4-thiadiazole derivatives $7(\mathbf{a}-\mathbf{o})$.

\begin{tabular}{|c|c|c|c|}
\hline Compound & $\mathrm{R}$ & Structure & M.p. $\left({ }^{\circ} \mathrm{C}\right)$ \\
\hline $7 a$ & & & $244-246$ \\
\hline $7 b$ & & & $136-138$ \\
\hline $7 \mathrm{c}$ & & & $170-172$ \\
\hline $7 d$ & & & 200-202 \\
\hline $7 e$ & & & $138-140$ \\
\hline $7 f$ & & & $228-230$ \\
\hline $7 g$ & & & $260-262$ \\
\hline
\end{tabular}


TABLe 1: Continued.

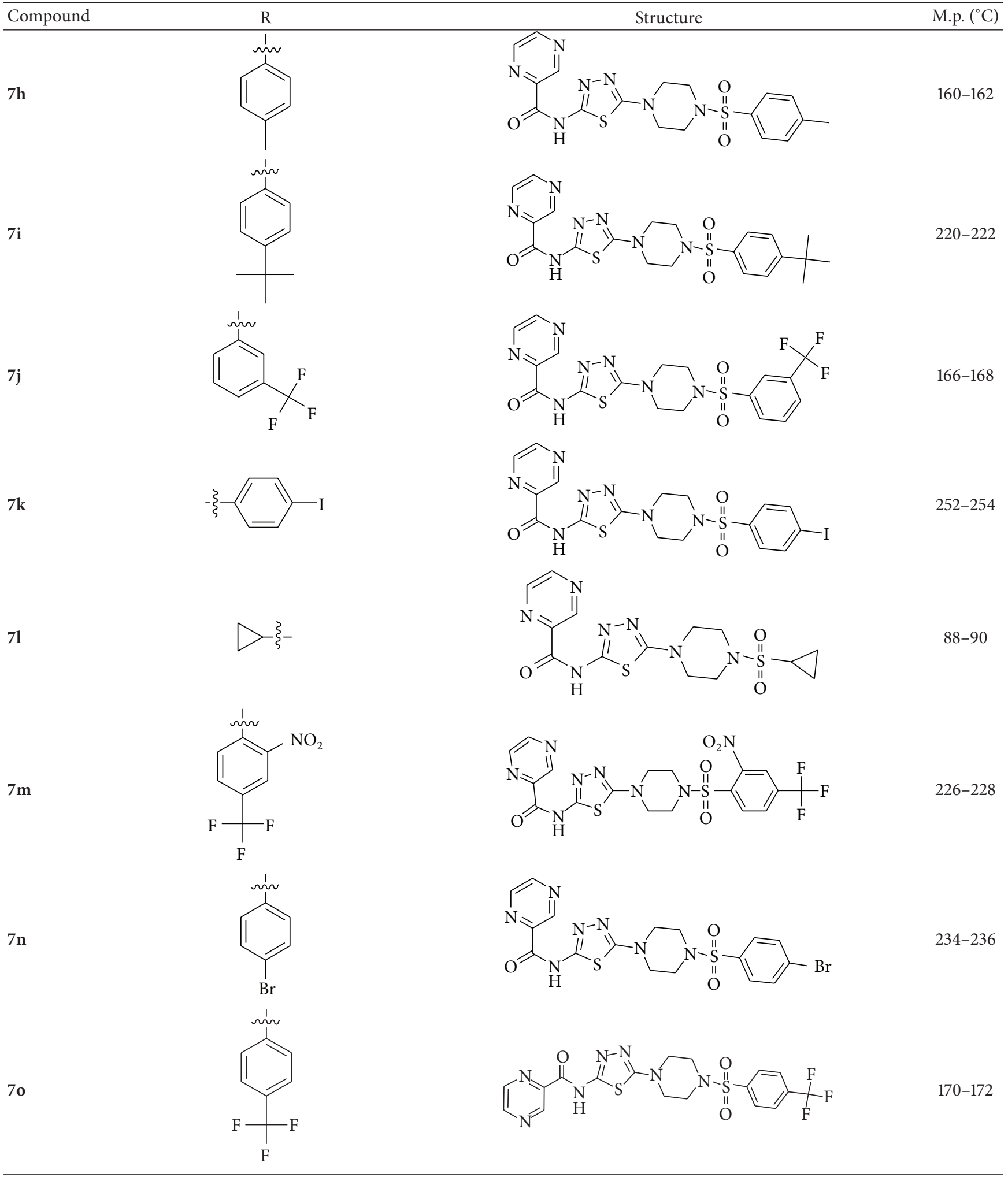

on male wistar rats. Seizures were induced in rats by delivering electro shock of $150 \mathrm{~mA}$ for $0.2 \mathrm{~s}$ by means of a convulsiometer through a pair of ear clip electrodes. The test compounds $(100 \mathrm{mg} / \mathrm{kg})$ were administered by oral route in the form of solution (the compounds were dissolved in $1 \%$ sodium carboxymethyl cellulose), 30 minutes before the maximal electroshock seizure test. The animals were observed closely for 2 minutes. The percentage of inhibition 


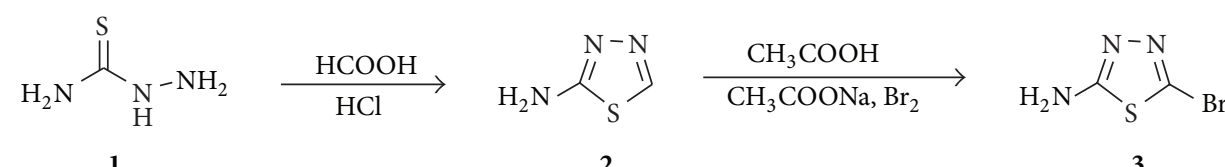

1

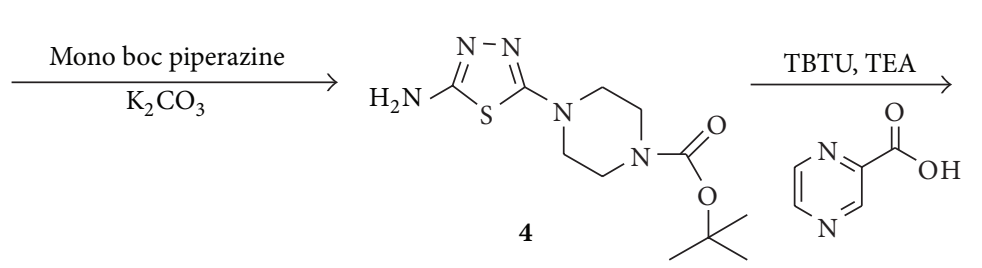

$\stackrel{4 \mathrm{~N} \mathrm{HCl} \text { in dioxane }}{\longrightarrow}$<smiles>CC(C)(C)OC(=O)N1CCN(c2nnc(NC(=O)c3cnccn3)s2)CC1</smiles><smiles></smiles>

$=\mathrm{H}, \mathrm{RSO}_{2}$

SCHEME 1

TABLE 2: Effect of compounds in the maximal electroshock seizure test.

\begin{tabular}{|c|c|c|}
\hline Treatment & $E / F$ & $\%$ Protection \\
\hline $7 \mathbf{a}$ & 6.51 & 48.70 \\
\hline $7 b$ & 7.38 & 37.20 \\
\hline $7 \mathrm{c}$ & 8.17 & 9.43 \\
\hline $7 d$ & 2.00 & 74.52 \\
\hline $7 e$ & 6.85 & 45.41 \\
\hline $7 f$ & 3.47 & 58.60 \\
\hline $7 \mathrm{~g}$ & 1.75 & 74.88 \\
\hline $7 \mathrm{~h}$ & 8.10 & 10.00 \\
\hline $7 \mathbf{i}$ & 8.05 & 10.62 \\
\hline $7 \mathbf{j}$ & 2.53 & 68.10 \\
\hline $7 \mathbf{k}$ & 4.70 & 50.31 \\
\hline 71 & 8.12 & 9.96 \\
\hline $7 \mathrm{~m}$ & 2.10 & 69.43 \\
\hline $7 n$ & 4.49 & 55.34 \\
\hline 70 & 2.96 & 65.20 \\
\hline Standard & 1.98 & 75.88 \\
\hline Control (vehicle) & 8.21 & - \\
\hline
\end{tabular}

$E / F=$ Extension/Flexion [Decrease in ratio of extension phase (in seconds)/flexion phase (in seconds)].

$\%$ Protection $=($ control-test $) /($ control $) * 100$.

of seizure relative to control was recorded and calculated [25]. Phenytoin $(100 \mathrm{mg} / \mathrm{kg})$ was used as a standard drug.

2.2.3. Neurotoxicity Screening. Acute neurological toxicity in mice was evaluated by rotarod test [25]. The mice were trained to stay on the accelerating rotarod that rotates at 10 revolutions per minute. The rod diameter was $3.2 \mathrm{~cm}$. Trained animals were administered with the test compounds
TABLE 3: Neurotoxicity screening of the compounds 7(a-o).

\begin{tabular}{|c|c|c|c|c|}
\hline \multirow{2}{*}{ Compound } & \multicolumn{4}{|c|}{ Neurotoxicity screen } \\
\hline & $0.5 \mathrm{~h}$ & $1 \mathrm{~h}$ & $2 \mathrm{~h}$ & $4 \mathrm{~h}$ \\
\hline $7 \mathbf{a}$ & $0 / 4$ & $0 / 4$ & $0 / 4$ & $0 / 4$ \\
\hline $7 b$ & $0 / 4$ & $0 / 4$ & $0 / 4$ & $0 / 4$ \\
\hline $7 \mathrm{c}$ & $0 / 4$ & $0 / 4$ & $1 / 4$ & $1 / 4$ \\
\hline $7 d$ & $0 / 4$ & $0 / 4$ & $0 / 4$ & $0 / 4$ \\
\hline $7 e$ & $0 / 4$ & $0 / 4$ & $0 / 4$ & $0 / 4$ \\
\hline $7 f$ & $0 / 4$ & $0 / 4$ & $0 / 4$ & $0 / 4$ \\
\hline $7 \mathrm{~g}$ & $0 / 4$ & $0 / 4$ & $0 / 4$ & $0 / 4$ \\
\hline $7 \mathrm{~h}$ & $0 / 4$ & $0 / 4$ & $1 / 4$ & $1 / 4$ \\
\hline $7 \mathbf{i}$ & $0 / 4$ & $0 / 4$ & $0 / 4$ & $0 / 4$ \\
\hline $7 \mathbf{j}$ & $0 / 4$ & $0 / 4$ & $0 / 4$ & $0 / 4$ \\
\hline $7 \mathbf{k}$ & $0 / 4$ & $0 / 4$ & $0 / 4$ & $0 / 4$ \\
\hline 71 & $0 / 4$ & $0 / 4$ & $1 / 4$ & $1 / 4$ \\
\hline $7 \mathrm{~m}$ & $0 / 4$ & $0 / 4$ & $0 / 4$ & $0 / 4$ \\
\hline $7 n$ & $0 / 4$ & $0 / 4$ & $0 / 4$ & $0 / 4$ \\
\hline 70 & $0 / 4$ & $0 / 4$ & $0 / 4$ & $0 / 4$ \\
\hline Standard & $0 / 4$ & $0 / 4$ & $0 / 4$ & $0 / 4$ \\
\hline
\end{tabular}

The data in the table represent the ratio between the numbers of the animals that exhibited neurotoxicity against the number of tested animals.

at the dose of $100 \mathrm{mg} / \mathrm{kg}$. Neurotoxicity was indicated by the inability of the animal to maintain equilibrium on the rod for at least one minute in each of the three trails. Phenytoin was used as a standard drug.

2.2.4. Statistical Analysis. In the present study, data were analyzed by one way analysis of variance (ANOVA) followed by dunnett test to compare difference between the groups. 


\section{Results and Discussion}

3.1. Chemistry. New pyrazine substituted 1,3,4-thiadiazole derivatives $7(\mathbf{a}-\mathbf{o})$ were synthesized according to Scheme 1. Readily available starting materials and simple synthesizing procedures make this method very attractive and convenient for the synthesis of various thiadiazoles. Formation of products was confirmed by recording their elemental analyses, ${ }^{1} \mathrm{H}$ NMR and mass spectra. The ${ }^{1} \mathrm{H}$ NMR, spectra of $7 \mathbf{g}$ showed piperazine ring in the region of $\delta, 3.31-3.88$. The mass spectra of $7 \mathrm{~g}$ showed molecular ion peak at $\mathrm{m} / z 566.4$ which is in agreement with the molecular formula, $\mathrm{C}_{19} \mathrm{H}_{15} \mathrm{~F}_{6} \mathrm{~N}_{7} \mathrm{O}_{3} \mathrm{~S}_{2}$. The elemental analyses data showed good agreement between the experimentally determined values and the theoretically calculated values within $\pm 0.4 \%$. The chemical structures and physical data of all the synthesized compounds are tabulated in Table 1.

3.2. Biological Activity. In the present study, the anticonvulsant activity of the synthesized compounds $7(\mathbf{a}-\mathbf{o})$ was evaluated by MES model at the dose of $100 \mathrm{mg} / \mathrm{kg}$ and the results are summarized in Table 2 . Compounds $7 \mathbf{d}$ and $7 \mathbf{g}$ demonstrated significant protective effect on MES induced seizure and the effect of $7 \mathbf{d}$ and $7 \mathrm{~g}$ was similar to that of standard (phenytoin). Similarly, compounds $7 \mathbf{f}, 7 \mathbf{j}, 7 \mathbf{k}$, $7 \mathbf{m}, 7 \mathbf{n}$, and $7 \mathbf{o}$ also showed good protective effect. Compounds $7 \mathbf{a}, 7 \mathbf{b}, 7 \mathbf{c}, 7 \mathbf{e}, 7 \mathbf{h}, 7 \mathbf{i}$, and $7 \mathbf{l}$ have relatively lower anticonvulsant potencies. All the compounds were examined for their neurotoxicity using rotarod method given in the dose of $100 \mathrm{mg} / \mathrm{kg}$. Except compounds $\mathbf{7 c}, \mathbf{7 h}$, and 7l, none of the compounds showed neurotoxicity. These compounds showed $25 \%$ toxicity compared to standard at $2 \mathrm{~h}$ of oral administration (Table 3).

The structural activity relationship study of these compounds indicate that the introduction of a piperazine group of pyrazine ring and 3,5-bis(trifluoromethyl) positions of the benzenesulfonyl moiety showed the best anticonvulsant activity in $\mathbf{7 g}$. Compound $\mathbf{7 d}$ contains halogen and thiophene group showed good anticonvulsant activity in the MES model. Both compounds did not exhibit neurotoxicity at the highest administered dose. Compounds with phenyl ring in $7 \mathbf{h}$ exhibited more anticonvulsant activity in comparison to methyl group (7c). Compounds $7 \mathbf{c}, 7 \mathbf{h}$, and $7 \mathbf{l}$ contribute $25 \%$ of neurotoxicity at $2 \mathrm{~h}$. Among the synthesized compounds $7(\mathbf{a}-\mathbf{o})$, all the compounds showed activity in the range of $9.43-74.88 \%$ in comparison to phenytoin which completely inhibited the convulsions produced by electroconvulsometer, but compound $\mathbf{7 g}$ having electron withdrawing groups showed excellent anticonvulsant activity.

\section{Conclusions}

In conclusion, a series of new pyrazine substituted 1,3,4thiadiazole derivatives $7(\mathbf{a}-\mathbf{0})$ were synthesized in good yield, characterized by different spectral studies, and their anticonvulsant activity have been evaluated. Various thiadiazole derivatives with electron withdrawing groups showed potent anticonvulsant activity. Among the synthesized compounds,
$7 \mathbf{d}$ and $7 \mathbf{g}$ showed excellent anticonvulsant activity. Compounds having thiadiazole ring showed better biological activities than compounds having oxadiazole ring.

\section{Conflict of Interests}

The authors declare that there is no conflict of interests regarding the publication of this paper.

\section{Acknowledgments}

The authors thank Dr. B. Veeresh, B. Madhava Reddy, and Nagula Naresh Kumar, Department of Pharmacology and Toxicology, G Pullareddy College of Pharmacy, Hyderabad, India, for carrying out Anticonvulsant activity.

\section{References}

[1] "Guidelines for epidemiologic studies on epilepsy. Commission on epidemiology and prognosis, international league against epilepsy," Epilepsia, vol. 34, no. 4, pp. 592-596, 1993.

[2] R. S. Fisher, W. van Emde Boas, W. Blume et al., "Epileptic seizures and epilepsy: definitions proposed by the international league against epilepsy (ILAE) and the international bureau for epilepsy (IBE)," Epilepsia, vol. 46, no. 4, pp. 470-472, 2005.

[3] World Health Organization, Aetiogy, Epidemiology and Prognosis, World Health Organization, Lyon, France, 2001.

[4] R. L. Krall, J. K. Penry, B. G. White, H. J. Kupferberg, and E. A. Swinyard, "Antiepileptic drug development-II: anticonvulsant drug screening," Epilepsia, vol. 19, no. 4, pp. 409-428, 1978.

[5] E. I. Isaacson, "Central nervous system depressants," in Wilson and Gisvold's Textbook of Organic Medicinal and Pharmaceutical Chemistry, J. N. Delgado and W. A. Remers, Eds., pp. 435-461, Lippincott-Raven, Philadelphia, Pa, USA, 1998.

[6] T. L. Lemke, D. A. Williams, V. F. Roche, and S. W. Zito, Foye's Principles of Medicinal Chemistry, Lippincott Williams \& Wilkins, Philadelphia, Pa, USA, 6th edition, 2007.

[7] W. Van Bebenburg, G. Steinmetz, and K. Thielek, "Substituierte polyaminopyridine," Chemiker-Zeitung, vol. 103, pp. 387-399, 1979.

[8] R. P. Shank, J. F. Gardocki, A. J. Streeter, and B. E. Maryanoff, "An overview of the preclinical aspects of topiramate: pharmacology, pharmacokinetics, and mechanism of action," Epilepsia, vol. 41, supplement 1, pp. S3-S9, 2000.

[9] M. Kubota, M. Nishi-Nagase, Y. Sakakihara et al., "Zonisamide-induced urinary lithiasis in patients with intractable epilepsy," Brain and Development, vol. 22, no. 4, pp. 230-233, 2000.

[10] J. A. Cramer, R. Fisher, E. Ben-Menachem, J. French, and R. H. Mattson, "New antiepileptic drugs: comparison of key clinical trials," Epilepsia, vol. 40, no. 5, pp. 590-600, 1999.

[11] S. W. Schneller and J. K. Luo, "Composition antiviral activity of substituted azaindoleoxoacetic piperazine derivative," Journal of Organic Chemistry, vol. 45, no. 20, pp. 4045-4048, 1980.

[12] O. Vitse, P. Bonnet, J. Bompart et al., "Nitration in the imidazo[1,2-a]pyrazine series. Experimental and computational results," Journal of Heterocyclic Chemistry, vol. 34, no. 3, pp. 701-707, 1997. 
[13] P. A. Bonnet, A. Michel, F. Laurent et al., "Synthesis and antibronchospastic activity of 8-alkoxy- and 8(alkylamino)imidazo[1,2-a]pyrazines," Journal of Medicinal Chemistry, vol. 35, no. 18, pp. 3353-3358, 1992.

[14] F. Azam, I. A. Ibn-Rajab, and A. A. Alruiad, "Adenosine A2A receptor antagonists as novel anti-Parkinsonian agents: a review of structure-activity relationships," Pharmazie, vol. 64, no. 12, pp. 771-795, 2009.

[15] I. Lalezari, A. Shafiee, A. Badaly et al., "Synthesis and pharmacological activity of 5 substituted 2 ( $N, N$ dialkylaminoethyl)amino and 2-N-methylpiperazinyl 1,3,4 thiadiazoles," Journal of Pharmaceutical Sciences, vol. 64, no. 7, pp. 1250-1252, 1975.

[16] P. Bhattacharya, J. T. Leonard, and K. Roy, "Exploring QSAR of thiazole and thiadiazole derivatives as potent and selective human adenosine $\mathrm{A}_{3}$ receptor antagonists using FA and GFA techniques," Bioorganic and Medicinal Chemistry, vol. 13, no. 4, pp. 1159-1165, 2005.

[17] M. R. Shiradkar, M. Ghodake, K. G. Bothara et al., "Synthesis and anticonvulsant activity of clubbed thiazolidinone-barbituric acid and thiazolidinone-triazole derivatives," Arkivoc, vol. 2007, no. 14, pp. 58-74, 2007.

[18] A. A. Ahmadu, A. U. Zezi, and A. H. Yaro, "Anti-diarrheal activity of the leaf extracts of Daniellia oliveri hutch and Dalz (Fabaceae) and ficus sycomorus Miq (Moraceae)," African Journal of Traditional, Complementary and Alternative Medicines, vol. 4, no. 4, pp. 524-528, 2007.

[19] M. V. Diurno, O. Mazzoni, G. Correale et al., "Synthesis and structure-activity relationships of 2-(substituted phenyl)-3-[3( $N, N$-dimethylamino)propyl]-1,3-thiazolidin-4-ones acting as H1-histamine antagonists," Il Farmaco, vol. 54, no. 9, pp. 579583, 1999.

[20] S. D. Firke, B. M. Firake, R. Y. Chaudhari, and V. R. Patil, "Synthetic and pharmacological evaluation of some pyridine containing thiazolidinones," Asian Journal of Research in Chemistry, vol. 2, pp. 157-161, 2009.

[21] T. Kato, T. Ozaki, and N. Ohi, "Improved synthetic methods of CP-060S, a novel cardioprotective drug," Tetrahedron Asymmetry, vol. 10, no. 20, pp. 3963-3968, 1999.

[22] D. Havrylyuk, B. Zimenkovsky, and R. Lesyk, "Synthesis and anticancer activity of novel nonfused bicyclic thiazolidinone derivatives," Phosphorus, Sulfur and Silicon and the Related Elements, vol. 184, no. 3, pp. 638-650, 2009.

[23] C. S. Andotra and B. S. Manhas, "Synthesis and antimicrobial activity of some 2,5-disubstituted 1,3,4-oxadiazoles," Acta Ciencia Indica, Series Chemistry, vol. 18, pp. 99-108, 1992.

[24] A. M. M. E. Omar and O. M. AboulWafa, "Synthesis and anticonvulsant properties of a novel series of 2-substituted amino-5-aryl-1,3,4-oxadiazole derivatives," Journal of Heterocyclic Chemistry, vol. 21, no. 5, pp. 1415-1418, 1984.

[25] H. G. Vogel and W. H. Vogel, Drug Discovery and Evaluation: Pharmacological Assays, vol. 2, Springer, Berlin, Germany, 1997. 

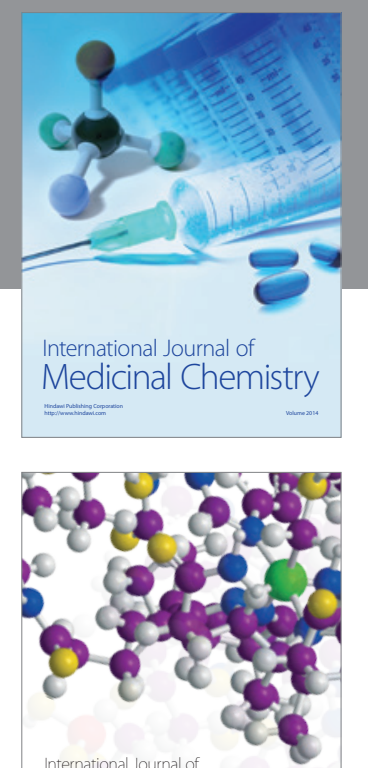

\section{Carbohydrate} Chemistry

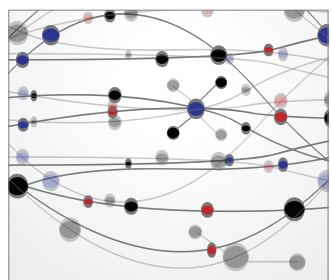

The Scientific World Journal
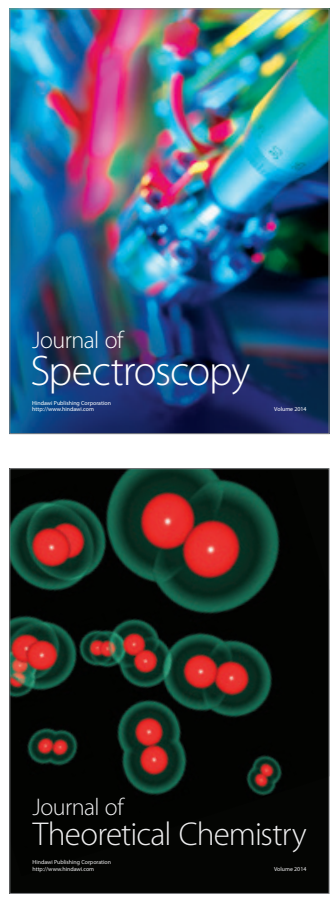
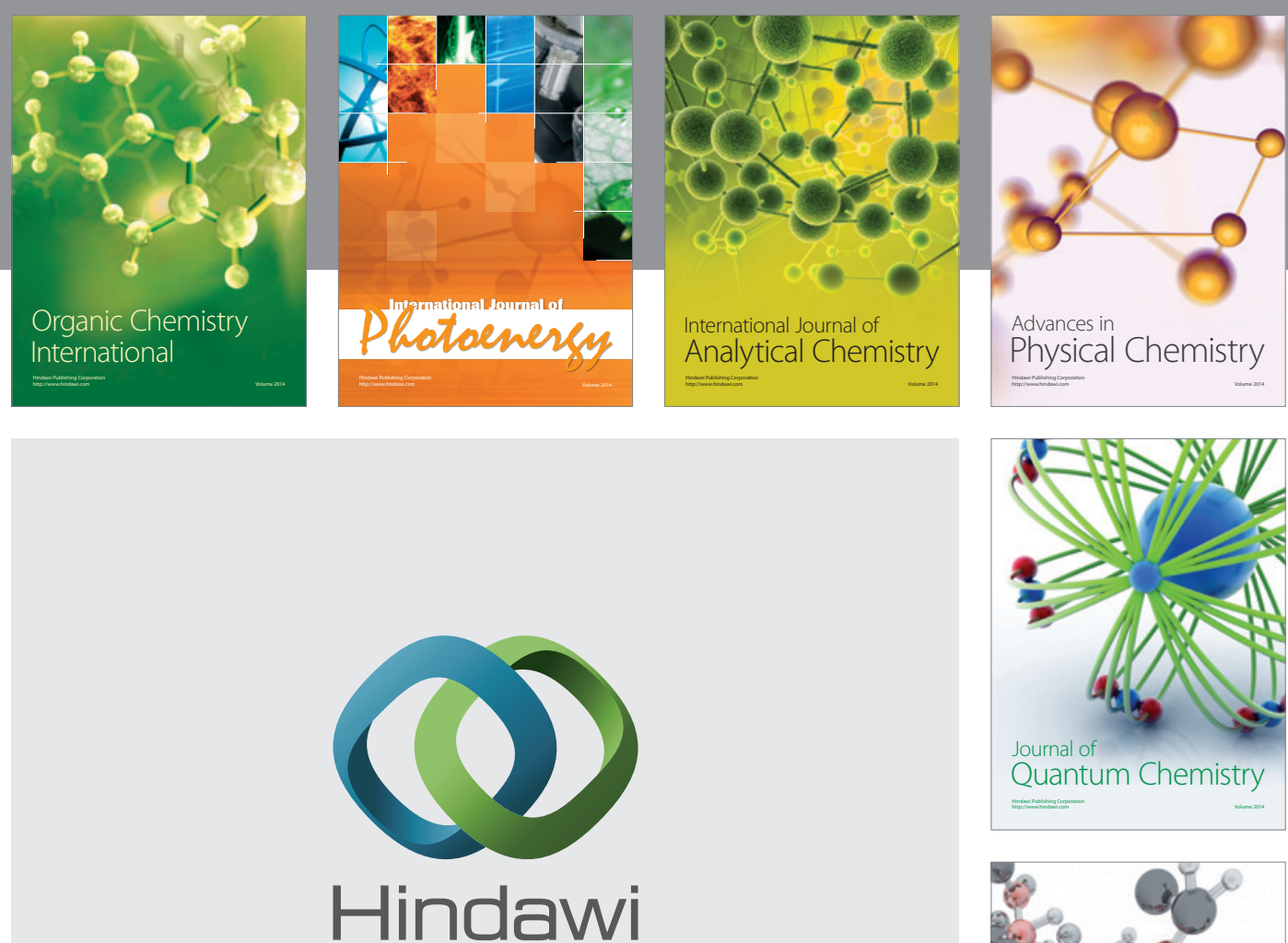

Submit your manuscripts at

http://www.hindawi.com

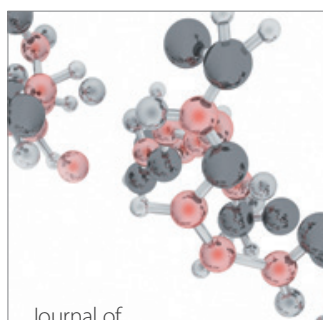

Analytical Methods

in Chemistry

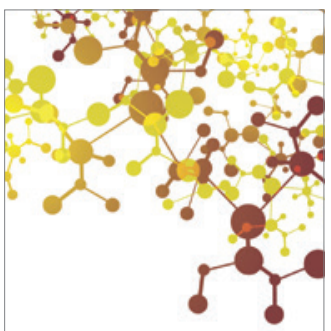

Journal of

Applied Chemistry

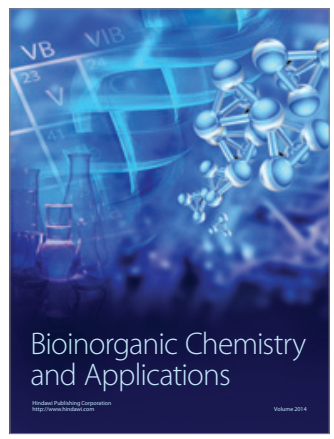

Inorganic Chemistry
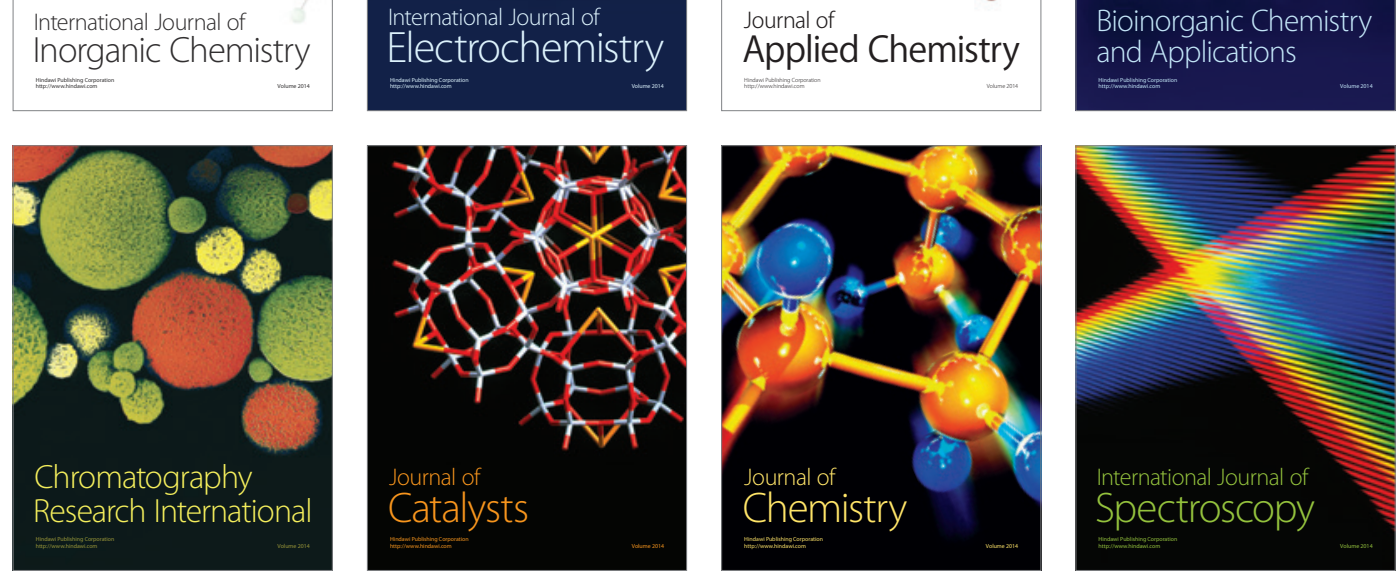